\title{
Maximum Likelihood Position Location with a Limited Number of References
}

\author{
D. Munoz-Rodriguez*¹, L. Suarez-Robles ${ }^{2}$, C. Vargas-Rosales ${ }^{3}$, J. R. Rodriguez-Cruz ${ }^{4}$ \\ 1,2,3,4 Department of Electrical and Computer Engineering, \\ ITESM-Monterrey. \\ Av. Eugenio Garza Sada 2501 Sur, Monterrey, \\ N.L., 64849, Mexico. \\ *dmunoz@itesm.mx
}

\begin{abstract}
A Position Location (PL) scheme for mobile users on the outskirts of coverage areas is presented. The proposed methodology makes it possible to obtain location information with only two land-fixed references. We introduce a general formulation and show that maximum-likelihood estimation can provide adequate PL information in this scenario. The Root Mean Square (RMS) error and error-distribution characterization are obtained for different propagation scenarios. In addition, simulation results and comparisons to another method are provided showing the accuracy and the robustness of the method proposed. We study accuracy limits of the proposed methodology for different propagation environments and show that even in the case of mismatch in the error variances, good PL estimation is feasible.
\end{abstract}

Keywords: Position location, maximum likelihood, wireless networks

\section{RESUMEN}

Se presenta un método de localización de la posición de dispositivos móviles en las fronteras de una región de cobertura. La metodología propuesta hace posible obtener la información de localización utilizando únicamente dos referencias fijas. Se introduce una formulación general y se muestra que la estimación de máxima verosimilitud puede proporcionar la información de localización de manera adecuada en este escenario. Se obtiene la caracterización del error de la posición por el valor rms del error y su distribución para diferentes escenarios de propagación. Además, a través de simulaciones y comparaciones con otros métodos, se muestra la exactitud y robustez de la metodología propuesta. Se estudian las limitaciones de exactitud de la metodología propuesta en diferentes ambientes de propagación y se muestra que aún en casos de diferencias en la varianza de los errores se obtiene una estimación de la posición con buena exactitud.

\section{Introduction}

Mobile network operators continuously seek new and innovative ways to create differentiation and to increase profits. One of the methods to accomplish these innovations is through the delivery of highly personalized services. One of the most powerful ways to personalize mobile services is to provide solutions based on location. In addition to Location Based Services (LBS), emergency services are also a plus for service providers. The main purpose of this paper is to introduce a methodology to carry out position location estimation when there are few fixed references (nontrilateration techniques) to begin with.
Position Location (PL) is an essential information tool to support law enforcement and emergency services, as well as being an enabler for proximity and location-based services. There are several location algorithm approaches, where PL is usually acquired through trilateration schemes involving range-estimation techniques based on fieldintensity measurements, time delay probes or angle-of-arrival observations. These approaches, in general, are supported by three or more references to obtain position location estimation. A discussion and the relative merits of these techniques can be found in [1], [2], [3], and [4]. In general, TDMA 
(Time Division Multiple Access) systems rely on time-delay observations associated with the propagation path between the Mobile Station (MS) and the $i^{\text {th }}$ Base Station, BSi. In the TDMA scenario, we can denote $t_{i}$ as the arrival time instant at BSi of a signal from the MS, $t_{0}$ and as the time at which the transmission by the MS took place, then the time-delay perceived at $\mathrm{BSi}$ becomes $\tau_{i}=t_{i}-t_{0}$.

In a fundamental scenario with multiple land-fixed references, we can consider that measurements are available in at least three BSs, and then the position location, within certain accuracy, can be estimated from the intersection of three circles whose radii are associated with the corresponding delays observed at those BSs. Since the transmission time reference $t_{0}$ is not generally available, it is convenient to consider the difference between the delay $\tau_{i}$ observed at BS and that observed at another base station, say at BS ${ }_{j}$, i.e., $\tau_{j}$, which results in $\varsigma_{i j}=\tau_{i}-\tau_{j}=t_{i}-t_{j}$, which is not dependent upon $t_{0}$, but on the relative position of the MS to the BSs. In this case, the location problem translates itself into an estimation of the intersection of two hyperboles.

In order to assist the PL estimation process, the Global System for Mobile Communications (GSM) suggests the deployment of conveniently placed Location Measurement Units (LMUs), [5]. Note that LMUs are meant to have limited resources and functionalities when compared to those of a BS, and yet they are able to provide timing information to the system which allows additional timedifference estimates. The placement of the LMU is left open to the service providers depending on their needs, and in this work, we propose to place the LMU at the edge of the network coverage area.

When a mobile is on the outskirts of a coverage area, (for instance in rural and suburban areas) the location problem becomes difficult because a trilateration process will not be necessarily possible to carry out due to the limited number of land-fixed references. This limitation precludes the adoption of alternative position-estimation schemes involving multiple redundant measurements, [6]. This is because the visibility of multiple BSs may not be viable for the intersection process, [1], [7]. In this paper, we show that the introduction of the LMU and its operation, together with a single BS and angle-of-arrival estimation, will allow the location of a subscriber on the outskirts of the coverage area.

This angle of arrival could be estimated using smart antennas. As a matter of fact, location may be inferred from the intersection of the hyperbola and the lines subtending the measured angles. We use the maximum-likelihood estimation (MLE) and also show that it provides adequate $\mathrm{PL}$ information. Performance of this estimation and comparison with MUSIC estimator as well as with the CramérRao Bound (CRB) is reported in the literature [8], where it was shown that the MLE is a good estimator, computationally speaking, even if a little accuracy is sacrificed. In contrast the MLE, asymptotically speaking, provides performance close to the CRB, thus, the MLE is sufficient to be considered as an efficient estimator for the location estimation problem. A general formulation is presented, and the Root Mean Square (RMS) error and error distribution are obtained for different propagation scenarios.

\section{Model Description}

In this section, we describe the fundamental procedure followed in our method, based on time difference and angle of arrival. We also discuss the issues concerning noisy measurements due to impairments in the environment. We, as well, introduce the solution methods used in the maximum likelihood formulation.

\subsection{Basic Scenario for PL}

The main scenario used in the methodology is shown in Figure 1. The methodology is based on the use of three parameters, i.e., time difference of arrival, angle of arrival at BS and angle of arrival at LMU. With these parameters or evidences, we carry out a mapping process between the subscriber location $(x, y)$ and the 3-dimensional surface given by those parameters. This is described as follows: 


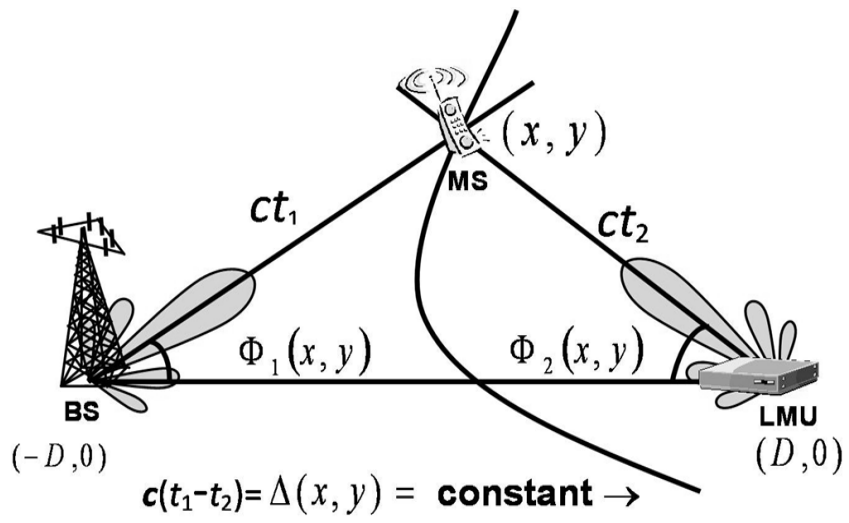

Figure 1. MS-LMU pair position location scheme.

Without loss of generality, let us assume a coordinate system with a BS located at $(-D, 0)$ and an LMU placed at $(D, 0)$, where $D$ is the cell's radius (see Figure 1). For this scenario, we use subscript 1 as a reference for the BS and subscript 2 as a reference for the LMU. Distance estimates are usually obtained through field intensity and travel time measurements; GSM favors the use of time observations (e.g., time advance measurements) since travel distances relate to propagation time by a constant.

For a mobile located at a point $\hat{\mathbf{p}}=(x, y)$, the propagation-time difference given by $\varsigma_{12}(x, y)=t_{1}-t_{2}$ corresponds, in a line-of-sight environment, to

$$
\begin{aligned}
& \Delta(x, y)=c \cdot \varsigma_{12}(x, y)=\sqrt{(D+x)^{2}+y^{2}} \\
& -\sqrt{(D-x)^{2}+y^{2}}
\end{aligned}
$$

where $c$ denotes the light's propagation speed and $\Delta(x, y)$ is the distance difference from the mobile to the BS and LMU. Since the BS and the LMU are assumed to be equipped with smart antennas, they also provide the angular information that relates location $(x, y)$, as follows:

$$
\begin{aligned}
& \Phi_{1}(x, y)=\operatorname{tg}^{-1}\left(\frac{y}{D+x}\right) \\
& \Phi_{2}(x, y)=\operatorname{tg}^{-1}\left(\frac{y}{D-x}\right) .
\end{aligned}
$$

Angular observations are taken with respect to the line joining the BS and the LMU; $\Phi_{1}(x, y)$ is the angle subtended by the BS-MS line, while is the angle subtended between the LMU-MS pair. Equations (1) to (3) enable us to obtain a surface mapping in the 3-dimensional space shown in Figure 2(a). For illustration purposes, the mapping of the straight trajectory in the $(x, y)$ plane joining points $(-1,1)$ and $(1,-1)$ is highlighted on the surface plots. Figure 2(b) presents the corresponding two dimensional projection on the $\left(\Phi_{1}(x, y), \Delta(x, y)\right)$ plane with the same trajectory highlighted. It is recognized that, in the absence of errors, the location can be solely obtained either from $\left(\Phi_{1}(x, y), \Phi_{2}(x, y)\right)$ or from $\left(\Phi_{i}(x, y), \Delta(x, y)\right)$, $i=1,2$. However, some ambiguities occur (for instance, when $\left.\Phi_{1}(x, y)=\Phi_{2}(x, y)=0\right)$. Furthermore, in the presence of the measurement estimation error, impairments cannot be reduced without additional observations. 

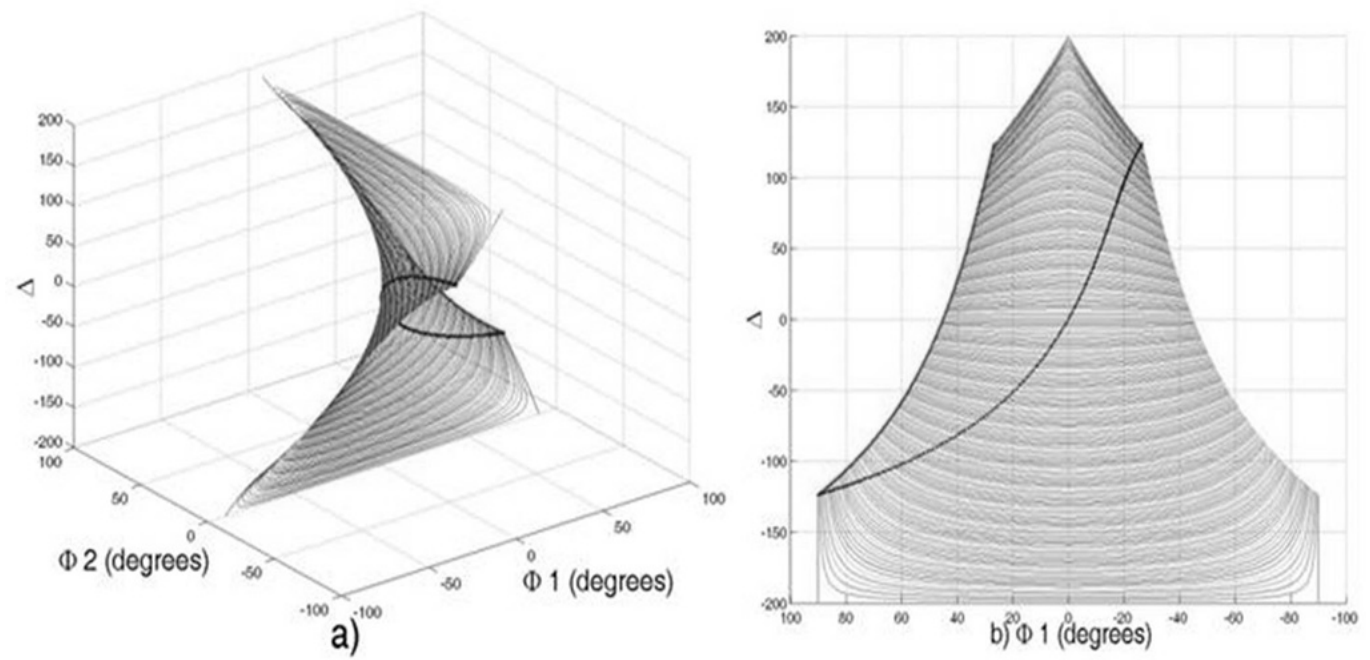

Figure 2. (a) 3D surface obtained from Equations (1), (2) and (3).

(b) 2-D projection corresponding to the $\left(\Delta, \Phi_{1}\right)$ plane.

\subsection{The Maximum Likelihood Model}

Note that the mapping obtained by using (1) to (3) is well behaved in the sense that there is a one-toone correspondence between the location $\hat{\mathbf{p}}=(x, y) \quad$ and the surface point $\hat{\pi}(x, y)=\left(\Phi_{1}(x, y), \Phi_{2}(x, y), \Delta(x, y)\right)$. In this way, the MS location $(x, y)$ could be obtained by conducting the inverse mapping $\left(\Phi_{1}, \Phi_{2}, \Delta\right) \rightarrow(x, y)$. In a real-life scenario, the observations are expected to be additively corrupted by propagation impairments. Thus, actual observations become noisy evidences given by $\quad \hat{\mathbf{\rho}}=\left(\rho_{1}, \rho_{2}, \rho_{3}\right)=\left(\Phi_{1}+\phi_{1}, \Phi_{2}+\phi_{2}, \Delta+\delta\right)$ where $\phi_{i}, \quad i=1,2$, and $\delta$ are measuring errors associated with the angular observations and timedifference measurements translated into distance differences, respectively. These errors are basically dependent upon the propagation conditions of the environment. Therefore, the estimated position $\left(x^{*}, y^{*}\right)$, i.e., the reverse mapping $\left(\rho_{1}, \rho_{2}, \rho_{3}\right) \rightarrow\left(x^{*}, y^{*}\right)$, may differ from the true position $(x, y)$. Even more, the observation vector $\hat{\rho}$ may not even lie on the $\left(\Phi_{1}(x, y), \Phi_{2}(x, y), \Delta(x, y)\right)$ surface, so, in this case, no inverse mapping can be directly conducted. Nevertheless, the inference problem is stated as follows: given an observation $\left(\rho_{1}, \rho_{2}, \rho_{3}\right)$, find the most probable surface point $\left(\Phi_{1}^{*}, \Phi_{2}^{*}, \Delta^{*}\right)$, and then command the inverse mapping $\left(\Phi_{1}^{*}, \Phi_{2}^{*}, \Delta^{*}\right) \rightarrow\left(x^{*}, y^{*}\right)$. Since the surface point $\hat{\pi}(x, y)=\left(\Phi_{1}(x, y), \Phi_{2}(x, y), \Delta(x, y)\right)$ is a function of the $(x, y)$ location, the optimal location-estimation problem can be stated as the appropriate selection of the coordinates $\left(x^{*}, y^{*}\right)$ in order to maximize the conditional probability density function of a mapping point $\hat{\pi}(x, y)$ given an evidence $\hat{\rho}$, i.e.,

$$
f\left(\hat{\pi}\left(x^{*}, y^{*}\right) \mid \hat{\mathbf{\rho}}\right)=\max _{(x, y)}\left\{\frac{f(\hat{\boldsymbol{\rho}} \mid \hat{\pi}(x, y)) f(\hat{\pi}(x, y))}{f(\hat{\mathbf{\rho}})}\right\} .
$$

For small noise variance, the solution $\left(x^{*}, y^{*}\right)$ to (4) is likely to be unique. However, for a very noisy 
evidence $\hat{\mathbf{\rho}}=\left(\rho_{1}, \rho_{2}, \rho_{3}\right)$, several optimal points could exist, making it necessary to disregard all solutions but one. This does not affect the average performance because this is a measure-zero event.

Although angular and delay observations may exhibit some correlation, [9], we assume, for simplicity, that the observation errors $\left(\phi_{1}, \phi_{2}, \delta\right)$ are statistically independent. Therefore, considering that the mobile can be located at any point with the same probability, the location problem is reduced to maximizing, with respect to $\hat{\pi}$, the likelihood function $f(\hat{\mathbf{p}} \mid \hat{\pi})$, [9]. Since measuring errors are assumed to be independent and to act additively, $f(\hat{\mathbf{\rho}} \mid \hat{\pi})$ can be written as follows:

$$
f(\hat{\rho} \mid \hat{\pi})=f_{\phi_{1}}\left(\rho_{1}-\Phi_{1}\right) \cdot f_{\phi_{2}}\left(\rho_{2}-\Phi_{2}\right) \cdot f_{\delta}\left(\rho_{34}-\Delta\right) .
$$

Actual angular and delay propagation distributions depend on mobile to base station separation distance and on characteristics as the scattering region width (srw) [11], [12]. Nevertheless, experimental results [12], [13], [14], [15] show that Laplacian and Gaussian distributions provide good descriptions for arrival angles, while time delays can adequately be approximated by an exponential distribution. Consequently, the delay difference $\delta$ can be considered to have a Laplacian distribution. Then, the likelihood function maximization can be stated as where $\sigma_{\phi}^{2}$ and $2 / \lambda_{\Delta}$ are, respectively, the angle variance and the distance difference variance associated with the scattering environment. Recall that the maximum likelihood estimator is more related to the mode location rather than to the distribution shape, and since the logarithm is a monotonic function, then the location $\left(x^{*}, y^{*}\right)$ that maximizes the likelihood function $f\left(\hat{\mathbf{\rho}} \mid \hat{\pi}\left(x^{*}, y^{*}\right)\right)$ will minimize the exponents in Equation (6).

\subsection{Solution Methods}

The closed form solution of (6) is cumbersome and several treatments can be followed. For instance, in order to reduce the formulation to a more common model, the Laplacian distribution behavior $\Delta \stackrel{d}{\approx} \frac{\lambda_{\Delta}}{2} e^{-\lambda_{\Delta}|\Delta|}$ can be approximated by a Gaussian distribution $\Delta \stackrel{d}{\approx} \frac{1}{\sqrt{2 \pi \sigma_{\Delta}}} e^{-\frac{\Delta^{2}}{2 \sigma_{\Delta}^{2}}}$ and it can be found that the best approximation in the Kullback-Leibler sense, [16], is achieved by equating $\sigma_{\Delta}^{2}=\left(\frac{1}{\lambda_{\Delta}}\right)^{2}$. This simplification together with the use of the maximum likelihood estimation allows having a wholly Additive Gaussian Model, which provides an estimator independent of the angular and distance difference variance.

A comparison of the proposed estimation mechanism to that of Torrieri's [6] is carried out. The measurement error in [6] is assumed to have a Gaussian distribution, and a Taylor series expansion is used to linearize the functions

$$
\begin{aligned}
& f\left(\widehat{\boldsymbol{\rho}} \mid \hat{\pi}\left(x^{*}, y^{*}\right)\right)=\max _{(x, y)}\left\{\frac{1}{2 \pi \sigma_{\varphi}^{2}} e^{-\frac{\left(\rho_{1}-t g^{-1}\left(\frac{y}{D+x}\right)\right)^{2}}{2 \sigma_{\varphi}^{2}}} e^{-\frac{\left(\rho_{2}-t g^{-1}\left(\frac{y}{D-x}\right)\right)^{2}}{2 \sigma_{\varphi}^{2}}}\right. \\
& \left.\frac{\lambda_{\Delta}}{2} e^{-\lambda_{\Delta}\left|c \rho_{3}-\sqrt{(D+x)^{2}+y^{2}}+\sqrt{(D-x)^{2}+y^{2}}\right|}\right\}
\end{aligned}
$$


specified by the exponents about a reference point $x_{0}$. This linearization results in a system of equations employed in the comparison presented in the Numerical Results Section of this paper. Alternatively, a linear approximation of (6), such as that in [17], could be used. However, in order to assess the optimal performance for a wide range of environments and have the needed accuracy, the solution $\left(x^{*}, y^{*}\right)$ is found by using an unconstrained nonlinear optimization method based on the BFGS (Broyden, Fletcher, Goldfarb and Shannon) Quasi-Newton algorithm, see [18], [19], [20] and [21], with the only constraint of having a continuous objective function. Since the surface $\hat{\pi}(x, y)$ is not linear, for very noisy evidences the solution might not necessarily be unique. Moreover, the obtained solution might be a local minimum therefore, minima discrimination is necessary in order to find such a point closest to the evidence. When several minima are of the same depth, one of these is randomly selected without affecting the average performance. Note that solution of (6) allows the estimation of $\left(x^{*}, y^{*}\right)$ in a form such that inverse mapping $\left(\Phi_{1}^{*}, \Phi_{2}^{*}, \Delta^{*}\right) \rightarrow\left(x^{*}, y^{*}\right)$ is not commanded explicitly but through the maximization of the likelihood function. Since MLE is a computationally efficient algorithm, its convergence time will be better than that using other estimators, [8].

\subsection{Performance perspective: The Stretching Factor}

A convenient form to assess the estimate $\left(x^{*}, y^{*}\right)$ is to compute its departure distance from the true $(x, y)$ location by using the Euclidean metric

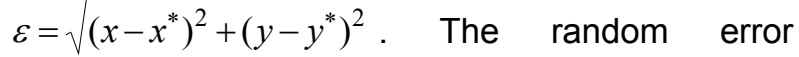
behavior will be governed by the propagation environment, characterized with the statistical parameters for angle $\sigma_{\phi}^{2}$, and for time difference $\sigma_{\Delta}^{2}$, and the location $(x, y)$ within the cell. Thus, root mean square (RMS) error $\sqrt{E\left\{\varepsilon^{2}\right\}}$ was adopted as a performance-analysis criterion.

In the literature, there exist many methods (tools) and manners to measure performance by considering certain aspects and perspectives of interest. Trying to get a general perspective from the point of view of location about the performance of the proposed scheme over the considered scenario, we consider the Stretching Factor (SF) as a performance tool. Performance is dependent both on angular and distance errors, as well as on the stretching factor defined by the mapping characteristics, (i.e., it depends also on the mobile's location). The mapping procedure plays an important role during this analysis because it reflects the effects of the movement of the MS over the observation space or surface mapping, where position estimations are made. The stretching factor provides a perspective on the performance of the proposed location method and the RMS in the different areas within the cell, as a measure of the stretching or rate of change of the mapping surface $S$ with respect to the movement of position of the MS during the mapping procedure defined by Equations (1) - (3). Important information about the direction and rate of change of such surface can be obtained from the gradient. It has an important geometric significance since it points on the direction in which the maximum rate of change occurs. In other words, the direction in which the mapping function increases most rapidly with respect to the MS displacement, while the magnitude of this function reflects the maximum rate of change.

The estimation robustness depends on the $\hat{\pi}(x, y)$ mapping's sensitivity to changes in $x$ and $y$. This dependence is often referred to in terms of a stretching factor $S_{f}$, [22], which relates how two close points in the $(x, y)$ plane may have distant projections on th $\hat{\pi}(x, y)=\left(\Phi_{1}(x, y), \Phi_{2}(x, y), \Delta(x, y)\right)$ space. A form of stretching factor definition is given by using the ratio of two corresponding areas in the and domains. This is

$$
S_{f}=\lim _{\delta \rightarrow 0} \frac{\operatorname{Area}\left\{S_{\delta(x, y)}\right\}}{\operatorname{Area}\left\{N_{\delta}(\hat{\mathbf{p}})\right\}},
$$

where $N_{\delta}(\hat{\mathbf{p}})$ is a $\delta$ neighborhood containing the point $\mathbf{p}=(x, y) . S_{\delta(x, y)}$ denotes the three-dimensional surface $S_{\delta(x, y)}=\left\{\left(\Phi_{1}(x, y), \Phi_{2}(x, y), \Delta(x, y)\right) \mid(x, y) \in N_{\delta}(\mathbf{p})\right\}$. 
In order to have a realistic average performance analysis, Monte Carlo simulations were conducted by assuming an evenly spread subscriber distribution throughout the coverage area of the cell.

\section{Numerical Results}

In order to evaluate the methodology, we simulated a scenario similar to that shown in Figure 1 with different propagation environments through the variation of the parameters $\sigma_{\phi}^{2}, \sigma_{\Delta}^{2}$. and We also varied the coverage radius of the cell, i.e., the separation of the LMU and BS.

The feasibility of the proposed algorithm was assessed via extensive simulation assuming a uniform distribution of users in the coverage area. Figures 3, 4 and 5 show simulation results of the RMS location error as the propagation distance error varies for a cell with radius of $1.5 \mathrm{~km}, 5 \mathrm{~km}$ and $10 \mathrm{~km}$, respectively.

As a way of comparison, we show results of the same scenario obtained by using our method proposed here, and the approximation method in [6]. The comparison was obtained by finding the estimate position given by the solution of Equation (14) in [6]. In order to do this comparison, we consider for the method in [6] a scenario where we have available the distance $D$, an angle of arrival, e.g., $\phi_{1}$, and a time difference. With these parameters, we obtain the initial estimation or reference point $x_{0}$ needed in the approximation of [6].

The figures show with solid lines the results obtained by using our method and with dotted lines the results provided by the solution of the approximation equations in [6]. In the figures, we can see how, for both methods, the RMS error increases when the angular error $\sigma_{\phi}$ grows. Also,degradation is seen when the distance error $\sigma_{\Delta}$ increases as well. We can also see that for large values of the distance error $\sigma_{\Delta}$, the maximum likelihood method proposed behaves more robustly compared to the approximation in [6]. For example, in Figure 3, we see that for a distance error $\sigma_{\Delta}$ above $200 \mathrm{~m}$, our method performs better than the linearization in [6], especially for the cases of $\sigma_{\phi}=2^{\circ}, 4^{\circ}, 6^{\circ}$. We can also see that for the case of $\sigma_{\phi}=2^{\circ}$, our method is better for all the values of the distance error $\sigma_{\Delta}$.

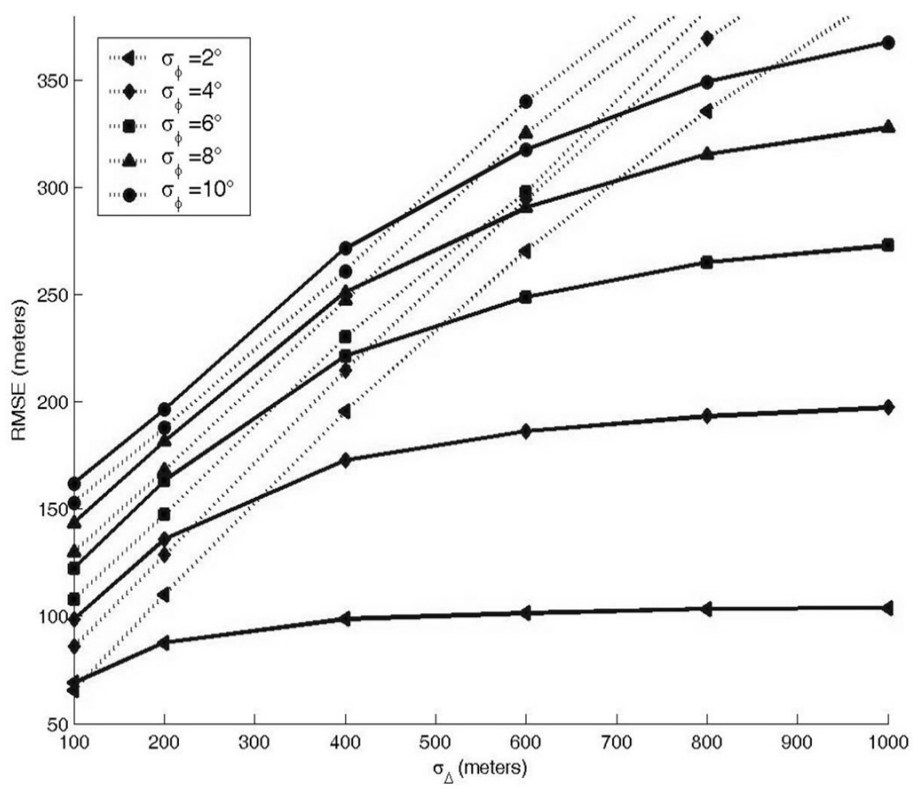

Figure 3. RMS error as delay spread $\sigma_{\Delta}$ varies in a $1.5 \mathrm{~km}$ cell. Method proposed in solid lines, method from [6] in dotted lines. 


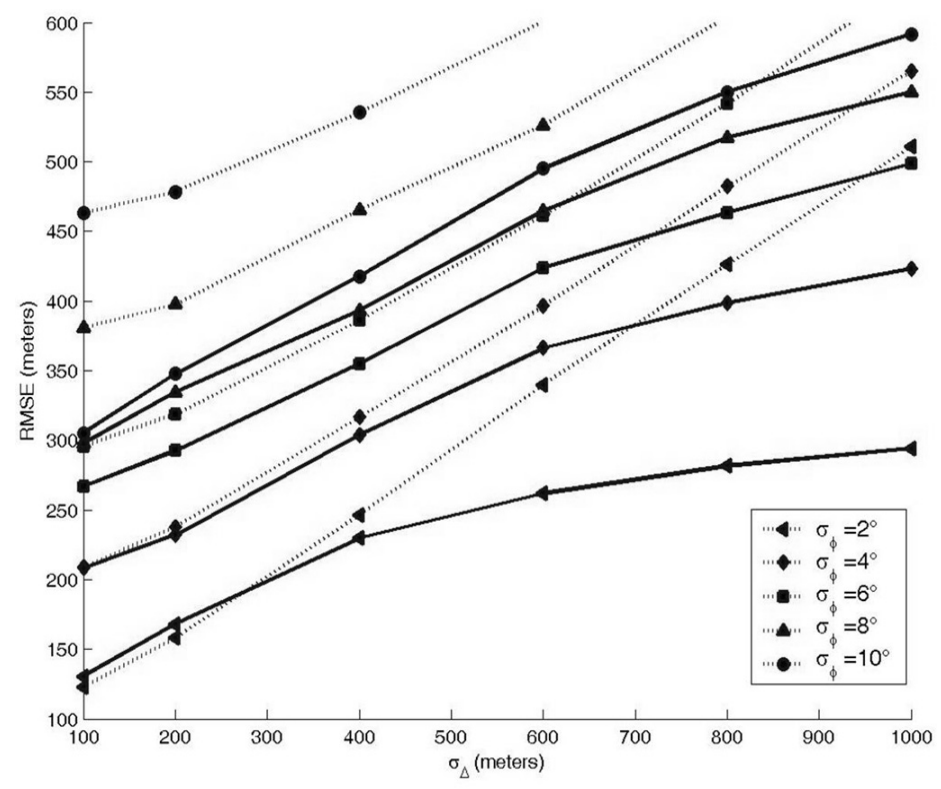

Figure 4. RMS error as delay spread $\sigma_{\Delta}$ varies in a $5 \mathrm{~km}$ cell. Method proposed in solid lines, method from [6] in dotted lines.

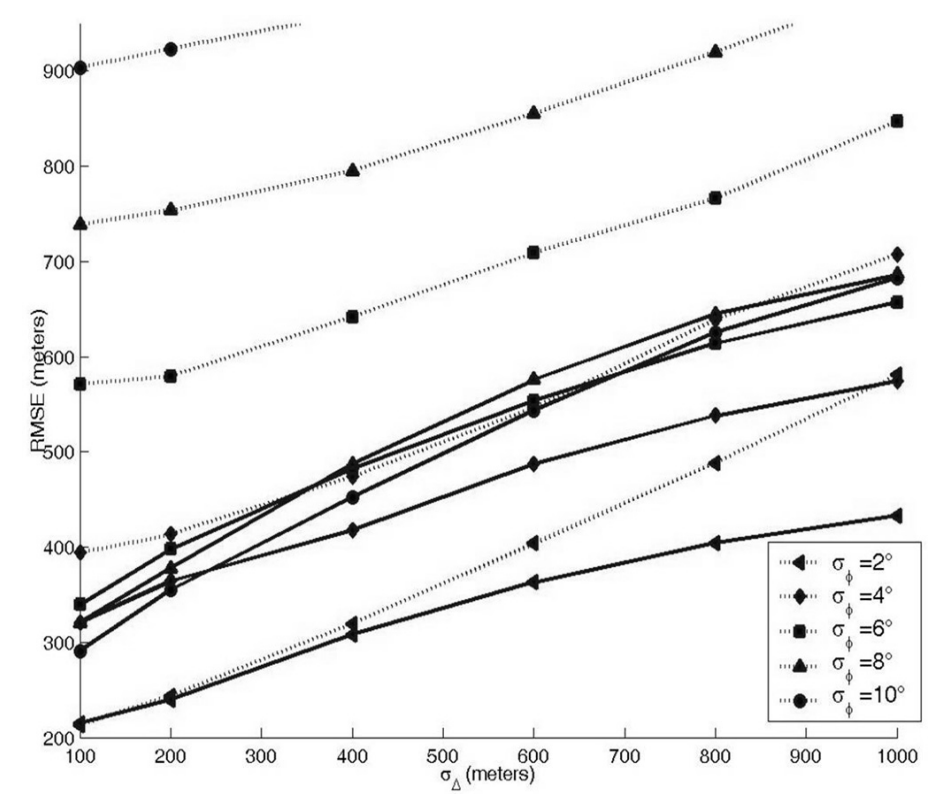

Figure 5. RMS error as delay spread $\sigma_{\Delta}$ varies in a $10 \mathrm{~km}$ cell. Method proposed in solid lines, method from [6] in dotted lines. 
By comparing the results obtained and shown in Figures 3,4 , and 5 , we can conclude that for small values of the distance error $\sigma_{\Delta}$, our method obtains better results than those from [6] as the cell radius increases. The same figures also show that the RMS error in our method does not grow as $\sigma_{\Delta}$ does in an apparent linear behavior, situation that the approximation in [6] shows. This behavior is due to the suboptimal solution in [6] by using the linearization of the problem, whereas our method utilizes nonlinear optimization of functions. Figures 4 and 5 show a similar behavior as that just described for both methods compared, but for larger cell radii.

Since goodness of location measurements is often referred to in terms of the proportion of cases when the error does not exceed a specified threshold, we include results for the probability that the error is below $100 \mathrm{~m}$ for different propagation environments in Figures 6 and 7. It can be observed that the obtained error is a function of the respective angular and time-difference standard deviations, i.e., the parameters $\left(\sigma_{\phi}, \sigma_{\Delta}\right)$. In Figures $6(a)$ and $7(a)$, we present the probability that the location estimation error is below $100 \mathrm{~m}$. We consider some values for the angular error such as $\sigma_{\phi}=2^{\circ}, 4^{\circ}, 6^{\circ}, 8^{\circ}, 10^{\circ}$ and vary the distance error $\sigma_{\Delta}$. We also indicate a typical value of the distance error for suburban environments on the figures. Through such figures, we can see the cases that satisfy the condition of an error location of $100 \mathrm{~m}$ or less, for $67 \%$ or more. In Figures $6(\mathrm{~b})$ and $7(b)$, we see similar results but with a variation on the angular error $\sigma_{\phi}$.

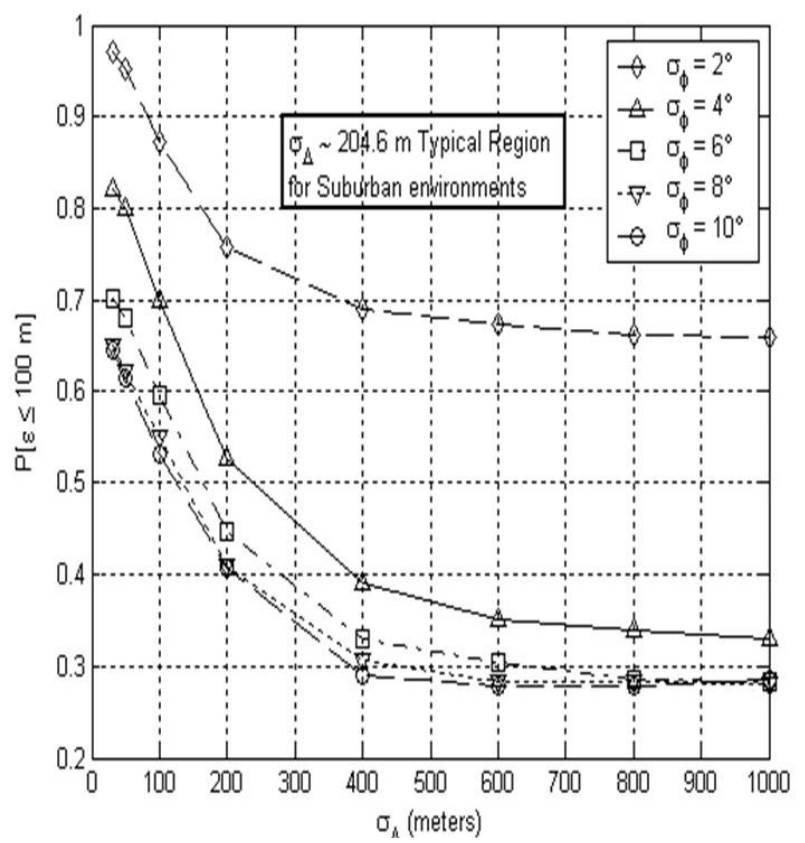

(a)

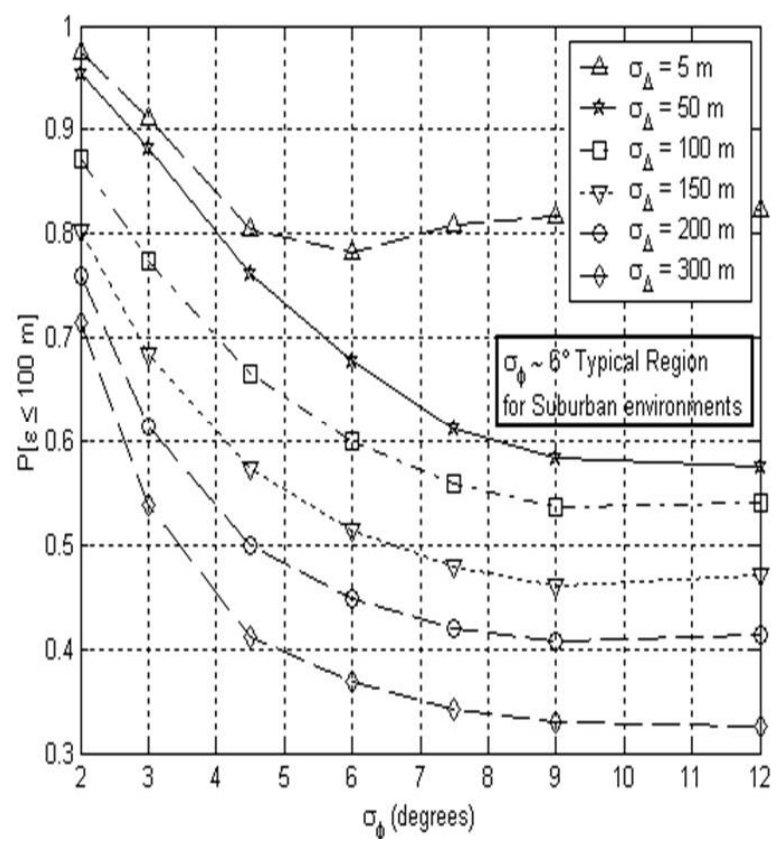

(b)

Figure 6. (a) Probability of location error smaller than 100 meters for $\sigma_{\phi}$ constant in a $1.5 \mathrm{~km}$ cell. (b) Probability of location error smaller than 100 meters for $\sigma_{\Delta}$ constant in a $1.5 \mathrm{~km}$ cell. 


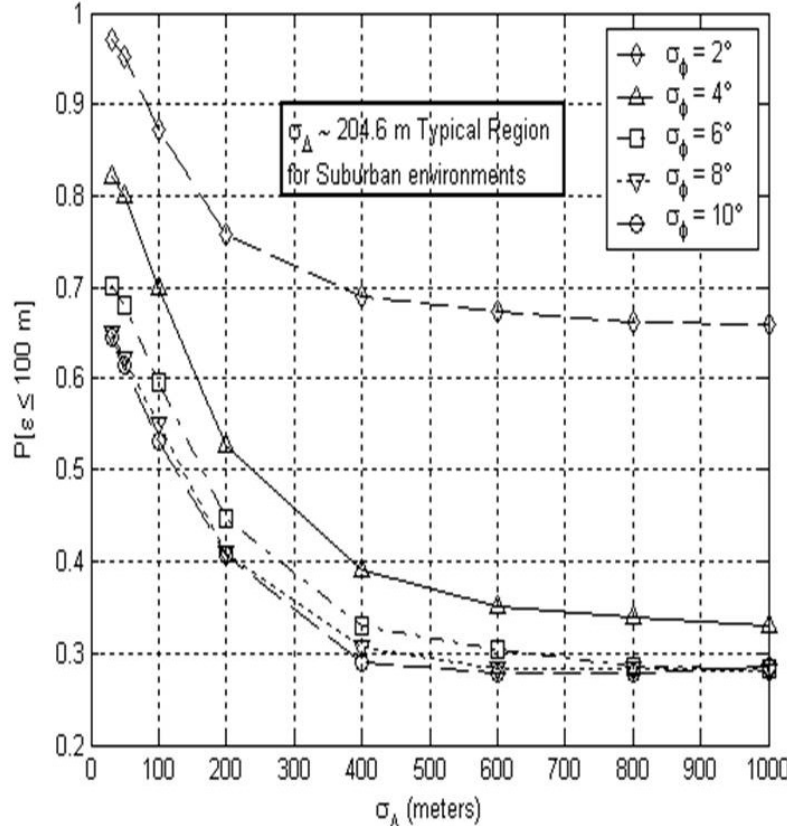

(a)

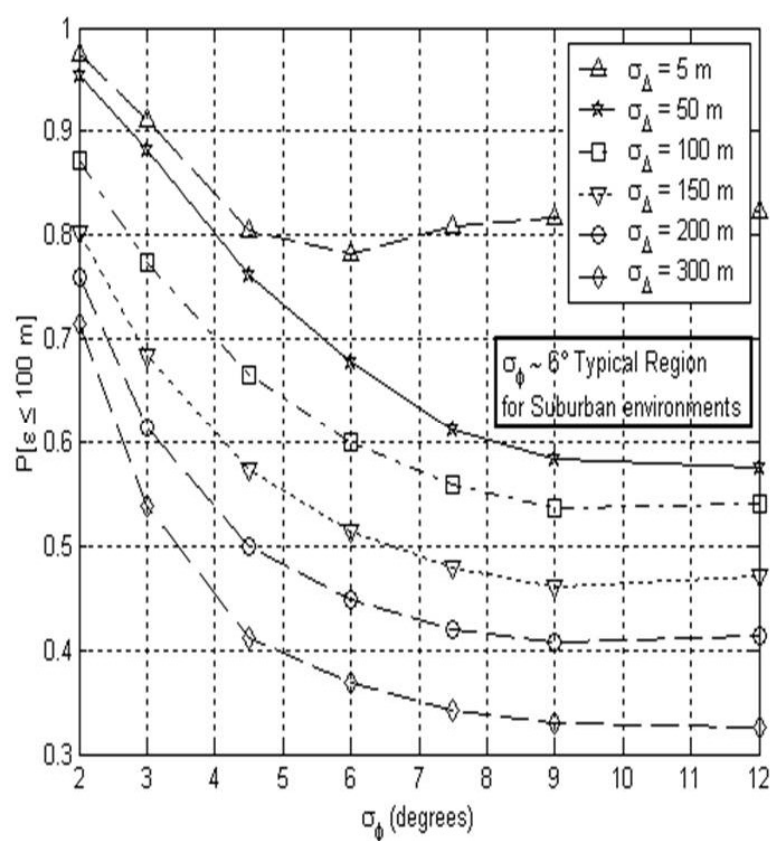

(b)

Figure 7. (a) Probability of location error smaller than 100 meters for $\sigma_{\phi}$ constant in a $5 \mathrm{~km}$ cell. (b) Probability of location error smaller than 100 meters for $\sigma_{\Delta}$ constant in a $5 \mathrm{~km}$ cell.

We know that in the case of a homogeneous Gaussian approximation, estimates become variance independent. Although variance estimation is not the main topic of this paper, some advantage may be taken by comparing measured distance difference and angular observation with the actual values associated to known location sites such as the BS and the LMU. These differences can be used to estimate the variance. We conduct a mismatch analysis by generating firstly a variance for the noise process in the environment that affects the observed parameters of measured distance difference and angular observation, and secondly, another variance that is used in the proposed method, thus generating a mismatch.
In order to statistically quantify the method's robustness, we carried out the mismatch analysis of the measured error variances byrunning simulations. The variances employed to estimate the position of the mobile were changed to differ from those employed to generate the noise, thus corrupting the evidences. Results show that the method introduced in this paper is robust to the mismatch where we obtained a difference in the RMS error of less than $2.5 \mathrm{~m}$ within a $1.5 \mathrm{~km}$ radius cell for a 20 percent mismatch in all variances, i.e., the parameters $\left(\sigma_{\phi}, \sigma_{\Delta}\right)$. This is shown in Figure 8. 


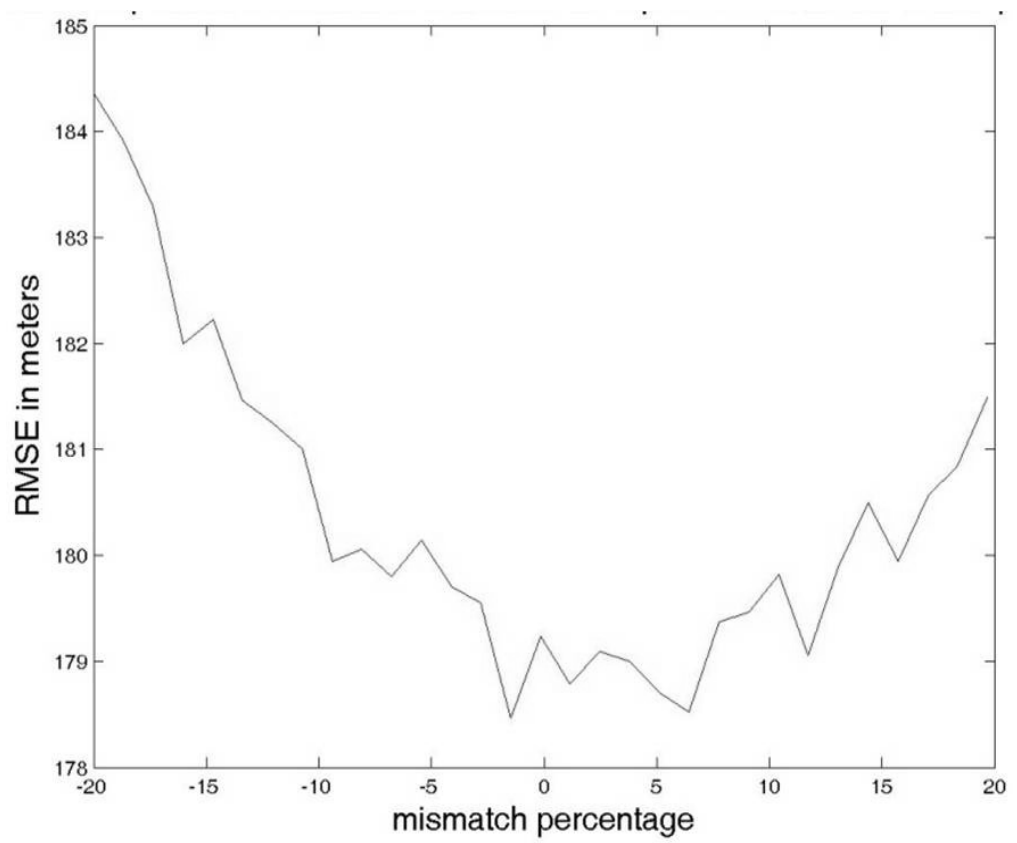

Figure 8. Relationship between RMS error and mismatch for a point in the center of the quadrant.

\section{Conclusions}

We have introduced a PL method for the outskirts of coverage areas. The method uses a nonlinear maximization of a multiparametric maximum likelihood function. We have compared this method to that in [6] and show that our method achieves good performance for a wide range of distance errors. Results show that an adequate position location is achieved with the proposed algorithm, relying only on two fixed references (BS and LMU). We also showed that the method is robust with respect to the error variances, showing small changes to the results as mismatch was increased. Although the proposed scheme has been initially intended for suburban/rural environments, the methodology can also be applied to urban environments. Accuracy of the scheme depends on the propagation delay and angular-spread characteristics.

\section{References}

[1] B. Ludden, et. al., Report on implementation issues related to access to location information by emergency services (E112) in the European Union, Final Report, Coordination Group on Access to Location Information by Emergency Services C.G.A.L.I.E.S., available from http://www.telematica.de/cgalies/ February 2002

[2] D. Munoz-Rodriguez, R. Estrada, C. Molina, and K. Basu, Cellular Position Location Techniques: a Parametric Detection Approach, Proceedings of the IEEE Vehicular Technology Conference, vol. 2, May 1999, pp. 1166-1171.

[3] Y. Zhao, Standardization of Mobile Phone Positioning for $3 G$ systems, IEEE Communications Magazine, vol. 40, No. 7 , July 2002, pp. 108-116.

[4] David Muñoz, Frantz Bouchereau, Cesar Vargas and Rogerio Enriquez, Position Location Techniques and Applications, 1st ed., Academic Press, 2009, pp. 23-100. 
[5] M. P. Wylie-Green and P. Wang, GSM Mobile Positioning Simulator, Proceedings of the IEEE Emerging Technologies Symposium: Broadband, Wireless Internet Access, April 2000, pp. 5.

[6] D.J. Torrieri, Statistical Theory of Passive Location Systems, IEEE Transactions on Aerospace and Electronic Systems, vol. 20, no. 2, March 1984, pp. 183198.

[7] I. Martin-Escalona, F. Barcelo, and J. Paradells, Delivery of Non-Standardized Assistance Data in EOTD/GNSS Hybrid Location Systems, Proceedings of The 13th IEEE International Symposium on Personal, Indoor and Mobile Radio Communications, vol. 5, September 2002, pp. 2347-2351.

[8] Stoica Petre and Nehorai Arie, MUSIC, Maximum Likelihood, and Cramér-Rao Bound: Further Results and Comparisons, IEEE Transactions on Acoustic, Speech and Signal Processing, vol. 38, no. 12, December 1990, pp. 2140-2150.

[9] R. B. Ertel and J. H. Reed, Angle and Time of Arrival Statistics for Circular and Elliptical Scattering Models, IEEE Journal on Selected Areas in Communications, vol. 17, no. 11, November 1999, pp. 1829-1840.

[10] D. J. Sakrison, Communication Theory: Transmission of Waveforms and Digital Information, John Wiley \& Sons, 1968

[11] A. Andrade and D. Covarrubias, Radio Channel Spatial Propagation Model for Mobile $3 G$ in Smart Antenna Systems, IEICE Transactions on Communications, vol. 8, 2003 pp. 213-220.

[12] A. Y. Olenco, K. T. Wong and M. Abdulla, Analytically Derived TOA-DOA Distributions of Uplink/Downlink Wireless-Cellular Multipaths arisen from Scatterers with an Inverted-Parabolic Spatial Distribution Around the Mobile, IEEE Signal Processing Letters, vol. 12 , No. 7, 2005, pp. 506-509.

[13] R. Janaswamy, Angle and Time of Arrival Statistics for the Gaussian Scatter Density Model, IEEE Transactions on Wireless Communications, vol. 1, no. 3, July 2002, pp. 488-497.

[14] W.C. Lee, Mobile Cellular telecommunications Systems, Mc.Graw-Hill, Singapore, 1989

[15] K. I. Pedersen, P. E. Mogensen, and B. H. Fleury, A Stochastic Model of the Temporal and Azimuthal Dispersion Seen at the Base Station in Outdoor Propagation Environments, IEEE Transactions on Vehicular Technology, vol. 49, no. 2, March 2000, pp. 437-447.
[16] S. Kullback and R. A. Leibler: On Information and Sufficiency, Ann. Math. Stat, 22, 1951, pp. 79-86.

[17] W. H. Foy, Position Location Solutions by Taylor Series Estimation, IEEE Transactions on Aerospace and Electronic Systems, vol. 12, 1976, pp. 187-194

[18] C.G. Broyden, The Convergence of a Class of Double-Rank Minimization Algorithms, Journal Inst. Math. Applic., vol. 6, 1970, pp. 76-90.

[19] R. Fletcher, A New Approach to Variable Metric Algorithms, Computer Journal, vol. 13, 1970, pp. 317322.

[20] D. Goldfarb, A Family of Variable Metric Updates Derived by Variational Means, Mathematics of Computing, vol. 24, 1970, pp. 23-26.

[21] D.F. Shannon, Conditioning of Quasi-Newton Methods for Function Minimization, Mathematics of Computing, vol. 24, 1970, pp. 647-656.

[22] J. M. Wozencraft and I. M. Jacobs, Principles of Communication Engineering, John Wiley \& Sons, 1965 


\section{Authors' Biographies}

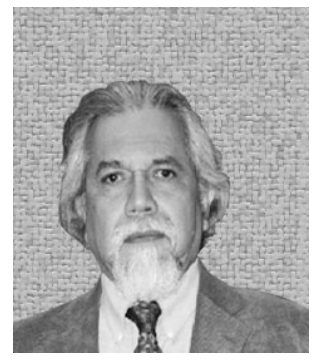

\section{David MUNOZ-RODRIGUEZ}

Doctor Munoz received the B.S. degree from the Universidad de Guadalajara, Guadalajara, Mexico in 1972, the M.S. degree from Cinvestav, Mexico City, Mexico in 1976, and the Ph.D. degree from University of Essex, Colchester, England in 1979, all of them in electrical engineering. He has been Chairman of the Communication Department and Electrical Engineering Department at Cinvestav, IPN. In 1992, he joined the Center for Electronics and Telecommunications at Instituto Tecnológico y de Estudios Superiores de Monterrey (ITESM), Campus Monterrey, Mexico, where he is the director. His research interests include transmission and personal communication systems.

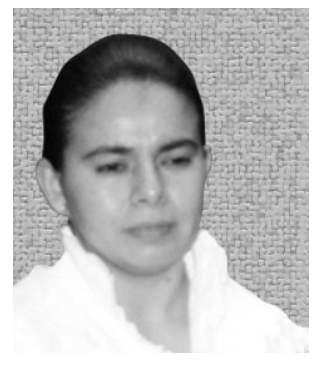

\section{Lluvia Gabriela SUAREZ-ROBLES}

She received the master of science in electronic engineering with specialization in telecommunications from ITESM- Campus Monterrey. She has contributed as a co-author of a position location patent.

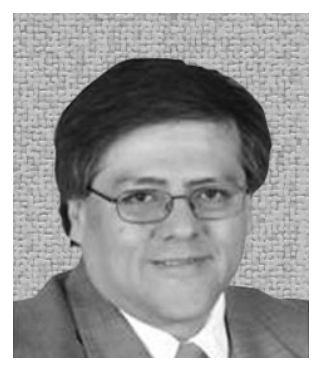

\section{Cesar VARGAS-ROSALES}

Dr. Vargas received a Ph.D. in electrical engineering from Louisiana State University in 1996. Thereafter, he joined the Center for Electronics and Telecommunications at Instituto Tecnológico y de Estudios Superiores de Monterrey (ITESM), Campus Monterrey, Mexico. He is currently the Telecommunications and Microelectronics Program Director at ITESM. Dr. Vargas is a member of the National System of Researchers (SNI) since 1997, and is the coauthor of the book Position Location Techniques and Applications. He has carried out research in the area of personal communication systems on CDMA, smart antennas, adaptive resource sharing, location information processing, and multimedia services. His research interests are personal communications networks, position location, mobility and traffic modeling, intrusion detection, and routing in reconfigurable networks. Dr. Vargas is the IEEE Communications Society Monterrey Chapter Head and has been a Senior Member of the IEEE since 2001. 


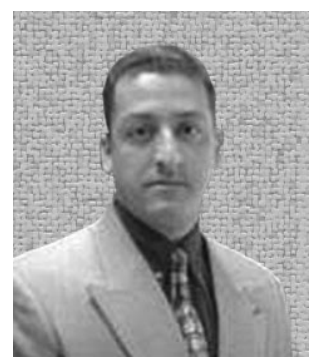

\section{Jose RAMON RODRIGUEZ}

Dr. Rodriguez received his Ph.D. in electrical engineering from CINVESTAVIPN, Zacatenco in August 2000. Thereafter, he joined the Center for Electronics and Telecommunications at Instituto Tecnológico y de Estudios Superiores de Monterrey (ITESM), Campus Monterrey, Mexico. He was a member of SNI for 8 years. He has participated in several government projects involving security and RF systems implementation. 